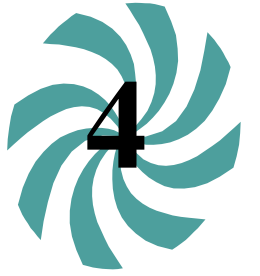

Tecnociencia, Vol. 23, $\mathrm{N}^{\circ} 1: 61-81$

Enero-Junio 2021

\title{
DESCRIPCIÓN MORFOMÉTRICA DE DOS POBLACIONES DE Leiosporoceros dussii (STEPHANI) HÄSSEL (LEIOSPOROCEROTACEAE) DE PANAMÁ.
}

\author{
${ }^{1}$ Guadalupe, Y. (D) ${ }^{1}$ Flores, N. (iD) $\&^{2,3}$ Villarreal, J. C. \\ ${ }^{1}$ Universidad de Panamá, Escuela de Biología, Departamento de Botánica. ${ }^{2}$ Instituto \\ Smithsonian de Investigaciones Tropicales. ${ }^{3}$ Universidad Laval, Departamento de \\ Biología. E-mail: yessenia27agb@gmail.com; naydaf@gmail.com; juan- \\ carlos.villarreal-aguilar@bio.ulaval.ca
}

\section{RESUMEN}

Leiosporoceros dussii es un antocerote neotropical que comprende colonias de cianobacterias alojadas en canales esquizógenos ramificados dicotómicamente a lo largo del talo, esta particularidad representa un carácter diagnostico utilizado para diferenciar esta especie de otros antocerotes. Este trabajo tiene como objetivo estudiar la variación morfológica de L. dussii, por medio de análisis morfométricos de dos poblaciones, ubicadas en El Valle de Antón, provincia de Coclé, Panamá. Se realizó una descripción completa de la especie y se encontraron variaciones morfométricas entre las dos poblaciones. Se compararon características morfométricas de las poblaciones estudiadas, las cuales mostraron variaciones morfométricas significativas, evidenciadas tanto en el análisis de datos multivariante como univariante. Este estudio proporciona descripciones morfométricas detalladas del gametófito y esporófito de $L$. dussii, las cuales pueden ser utilizadas para la identificación de especímenes sin estructuras reproductivas.

\section{PALABRAS CLAVES}

Plantas no vasculares, Antocerote, gametófito, esporófito, morfometría, característica morfológica. 


\title{
MORPHOMETRIC DESCRIPTION OF TWO POPULATIONS OF Leiosporoceros dussii (STEPHANI) HÄSSEL (LEIOSPOROCEROTACEAE) IN PANAMA.
}

\begin{abstract}
Leiosporoceros dussii (Stephani) Hässel is a neotropical genus of hornwort with little exiting morphological information. This is the only hornwort reported with cyanobacterial colonies present in longitudinally oriented schizogenous canals through the thallus, which is a diagnostic feature for its identification. The aim of this study was to describe the morphology of $L$. dussii using morphometrical analysis of two populations from El Valle de Anton, in the Cocle Province of Panama. Our analysis showed significant variations in both the gametophyte and the sporophyte when the two populations were compared using multivariate and univariate statistical analysis. This description is a valuable source for the identification of sterile plants of the species Leiosporoceros dussii and expand our knowledge of this phylogenetically important species.
\end{abstract}

\section{KEY WORDS}

Non-Vascular Plants, hornwort, gametophyte, sporophyte, morphometric, morphological characteristic.

\section{INTRODUCCIÓN}

Las briofitas en sentido amplio, comprende tres líneas monofiléticas de plantas no vasculares: Marchantiophyta (hepáticas), Anthocerotophyta (antocerotes) y Bryophyta (musgos), lo cual se evidenció en estudios del origen y evolución de los estomas, estructuras presentes tanto en plantas no vasculares como en plantas vasculares (One Thousand Plant Transcriptomes Initiative 2019). Representan el segundo grupo de plantas más diverso, después de las plantas con flores (Konrat et al. 2010). Los antocerotes constituyen el grupo menos abundante entre las plantas no vasculares y actualmente comprende unas 200-250 especies (Villarreal et al. 2010), lo que corresponde a un número pequeño en comparación con los musgos (Magill 2010) y hepáticas (Von Konrat et al. 2014). 
Los antocerotes poseen una morfología interesante, muchas de las especies de este grupo presentan unas estructuras singulares semejantes a los pirenoides de las algas verdes, las cuales son consideradas como estructuras análogas, debido a la similitud en la cantidad y ubicación de la enzima RuBIsCO en forma activa (Vaughn et al. 1990). Además, los antocerotes presentan estomas en la superficie del esporofito, los cuales a pesar de ser idénticos a los estomas encontrados en las angiospermas, se consideran estructuras análogas, debido a que no poseen el mismo patrón de división celular (Evert 2016), ni las mismas funciones (Pressel et al. 2014). Por otro lado, la presencia de un meristema basal en el esporofito, con meiosis asincrónica, es una característica exclusiva de los antocerotes (Villarreal \& Renzaglia 2015). Los antocerotes además de poseer una compleja morfología, posiblemente, representan el linaje de plantas más antiguo de la tierra, con una amplia capacidad de desarrollar simbiosis con cianobacterias (Villarreal \& Renzaglia 2015).

Leiosporoceros dussii (Stephani) Hässel es una especie neotropical, fue descrita originalmente como Anthoceros dussii Stephani. por Stephani en 1893. Luego en 1916 éste la describe como múltiples especies del género Aspiromitus Stephani. En 1986, Gabriela G. Hässel de Menéndez propone el género monotípico Leiosporoceros Hässel, en el cual incluye a Leiosporoceros dussii (Stephani) Hässel. Este taxón se distingue de los demás miembros de la división Anthocerotophyta por el arreglo bilateral alterno-opuesto de sus esporas. Esta característica fue importante para establecer la familia Leiosporocerotaceae. La presencia de las hebras de Nostoc en los canales esquizógenos es otra característica única que separa a Leiosporoceros de otros antocerotes y representa un arreglo simbiótico nuevo en plantas terrestres (Villarreal \& Renzaglia 2006) Todas estas descripciones antes mencionadas están basadas en las características del esporofito (esporas y eláteres), con una breve descripción del gametófito.

Sorprendentemente, los canales de Nostoc en Leiosporoceros han sido desapercibidos o reportados como grandes colonias globosas en estudios previos (Stephani 1893; de Menéndez 1986); por lo tanto, existe una necesidad de realizar estudios adicionales sobre el gametófito de antocerotes con material fresco, ya sea a partir de cultivos axénicos de laboratorio o recolectados de campo. El estudio del gametófito de estas 
plantas podría ampliar las características útiles para la identificación de estos organismos, las cuales están actualmente casi restringidas a las características de la morfología del esporófito. Villarreal \& Renzaglia (2006), realizaron un estudio sobre el desarrollo de las colonias de cianobacterias dentro del talo de Leiosporoceros, en donde aportan información relacionada a la morfología del gametófito. Sin embargo, no se ha estudiado la variación morfológica de L. dussii en diferentes poblaciones. El objetivo de este estudio es realizar una descripción morfológica de la especie Leiosporoceros dussii (Stephani) Hässel y determinar sus variaciones, tanto del gametófito como del esporófito, mediante análisis morfométricos de dos poblaciones.

\section{METODOLOGÍA}

Recolección y preparación de las muestras:

Las plantas utilizadas en este estudio se tomaron de dos poblaciones pequeñas ubicadas en El Valle de Antón, en Río Indio y en Río Guayabo. De cada población se utilizaron dos colonias, una femenina y una masculina; de cada colonia se utilizaron cinco talos para realizar las mediciones correspondientes al análisis morfométrico (Cuadro 1). Las colonias de plantas fueron recolectadas del suelo con una navaja de bolsillo y colocadas en bolsas con cierre hermético debidamente rotuladas. Luego de ser extraídas del suelo, las plantas recolectadas se guardaron en un recipiente refrigerado con hielo para mantenerlas frescas hasta ser procesadas en el laboratorio. Para tomar las medidas macroscópicas y hacer cortes del talo, se utilizó un estereomicroscopio modelo Leica: ZOOM 2000 y una regla milimetrada. Las fotografías de estructuras microscópicas fueron tomadas utilizando un microscopio de luz modelo OLYMPUS: BX50 con cámara fotográfica OLYMPUS: DP25. Posteriormente, las estructuras captadas en las fotografías fueron analizadas y medidas con el software DP2-BSW OLYMPUS.

Especímenes examinados:

Panamá. Provincia de Coclé: El Valle de Antón, Río Guayabo, dentro del Monumento Natural Cerro Gaital, sobre ladera con suelo de roca volcánica, 8³7'34.6”N, 8008'13.7’O, 624 m. Guadalupe 240 y Guadalupe 241 (PMA); Río Indio, dentro del Monumento Natural Cerro

Gaital, sobre ladera con suelo de roca volcánica, $8^{\circ} 38^{\prime} 25.5^{\prime} \mathrm{N}$, 8006'49.2”O, Guadalupe 247 y Guadalupe 248 (PMA).

Análisis morfométrico: 
Se consideraron 13 variables del gametófito y 9 variables del esporófito (Cuadro 1). Se compararon las variables en cuatro grupos con cantidad de medidas similares con una prueba multivariante MANOVA para determinar si existen diferencias significativas entre las dos poblaciones estudiadas dentro de cada grupo (Cuadro 2). De igual manera se realizó un análisis de componentes principales (PCA) de cada grupo, los cuales fueron representados en gráficas biplot. Además, se analizaron los datos de las variables de manera independiente con la prueba paramétrica $t$ student y la prueba no paramétrica Mann-Whitney, según su normalidad de varianza, para comparar las dos poblaciones y así determinar qué variables muestran significancia en las diferencias entre las dos poblaciones de manera independiente. Se hizo una gráfica boxplot para mostrar la comparación de la cantidad de anteridios entre las dos poblaciones. Todos los análisis morfométricos fueron realizados con el programa PAST, a excepción de los cálculos de la prueba MANOVA, los cuales fueron realizados con el programa de análisis estadístico R 3 . 5. 1. (R Core Team, 2018). 
Cuadro 1. Variables Morfométricas. Se detallan las mediadas realizadas para el análisis morfométrico por una población estudiada según el género de las plantas.

\begin{tabular}{|c|c|c|c|}
\hline \multirow{2}{*}{$\begin{array}{c}\text { VARIABLE } \\
\text { MORFOMÉTRICA }\end{array}$} & \multicolumn{3}{|c|}{ MEDIDAS POR POBLACIÓN } \\
\hline & $\begin{array}{l}\text { Tipo De } \\
\text { Planta }\end{array}$ & Medidas Por Talo & $\begin{array}{l}\text { Medidas } \\
\text { Por } \\
\text { Población }\end{array}$ \\
\hline \multicolumn{4}{|l|}{ GAMETÓFITO } \\
\hline Ancho del talo & $\hat{\sigma} / q$ & una por talo & 10 \\
\hline Largo del talo & $\pi / q$ & una por talo & 10 \\
\hline $\begin{array}{l}\text { Ancho de células } \\
\text { interiores }\end{array}$ & $\hat{\sigma} / q$ & cinco células por talo & 25 \\
\hline $\begin{array}{l}\text { Largo de células } \\
\text { interiores }\end{array}$ & $\hat{o} / q$ & cinco células por talo & 25 \\
\hline $\begin{array}{l}\text { Ancho de células } \\
\text { epidermales }\end{array}$ & $\delta / q$ & cinco células por talo & 25 \\
\hline $\begin{array}{l}\text { Largo de células } \\
\text { epidermales }\end{array}$ & $\delta / q$ & cinco células por talo & 25 \\
\hline $\begin{array}{l}\text { Ancho de } \\
\text { cloroplastos }\end{array}$ & $\delta / q$ & $\begin{array}{l}\text { cinco cloroplastos por } \\
\text { talo }\end{array}$ & 25 \\
\hline Largo de cloroplastos & $\delta / q$ & $\begin{array}{l}\text { cinco cloroplastos por } \\
\text { talo }\end{array}$ & 25 \\
\hline $\begin{array}{l}\text { Cantidad de } \\
\text { anteridios por cámara } \\
\text { Anteridial }\end{array}$ & $\hat{\sigma}$ & tres cámaras por talo & 15 \\
\hline Ancho del anteridio & $\hat{\delta} / q$ & $\begin{array}{l}\text { cinco por cámara } \\
\text { anteridial }\end{array}$ & 75 \\
\hline Largo del anteridio & $\hat{\jmath} / q$ & $\begin{array}{l}\text { cinco por cámara } \\
\text { anteridial }\end{array}$ & 75 \\
\hline $\begin{array}{l}\text { Ancho del pedicelo } \\
\text { del anteridio }\end{array}$ & $\delta / q$ & $\begin{array}{l}\text { cinco por cámara } \\
\text { anteridial }\end{array}$ & 75 \\
\hline $\begin{array}{l}\text { Largo del pedicelo } \\
\text { del anteridio }\end{array}$ & $\hat{\sigma} / q$ & $\begin{array}{l}\text { cinco por cámara } \\
\text { anteridial }\end{array}$ & 75 \\
\hline \multicolumn{4}{|l|}{ ESPORÓFITO } \\
\hline Largo del esporófito & q & una por talo & 10 \\
\hline $\begin{array}{l}\text { Diámetro del } \\
\text { esporófito }\end{array}$ & q & una por talo & 10 \\
\hline Largo del involucro & q & una por talo & 10 \\
\hline $\begin{array}{l}\text { Grosor de tejido } \\
\text { asimilativo }\end{array}$ & q & una por esporófito & 10 \\
\hline Ancho de las esporas & q & diez por talo & 50 \\
\hline
\end{tabular}




\begin{tabular}{|l|l|l|c|}
\hline Ancho del estoma & + & diez por talo & 50 \\
\hline Ancho del ostiolo & + & diez por talo & 50 \\
\hline $\begin{array}{l}\text { ancho de células de } \\
\text { los estomas }\end{array}$ & + & diez por talo & 50 \\
\hline $\begin{array}{l}\text { largo de células de } \\
\text { los estomas. }\end{array}$ & + & diez por talo & 50 \\
\hline
\end{tabular}

Cuadro 2. Lista de Abreviaciones. Se detallan el nombre de cada tipo de variable morfométrica realizada con su correspondiente abreviatura utilizadas en las gráficas biplot del Análisis de Componentes Principales (PCA). Los colores representan los grupos de variables utilizados en las pruebas de análisis multivariantes. Azul: Grupo A, Rojo: Grupo B, Verde: Grupo C y Naranja: Grupo D.

\begin{tabular}{|c|c|c|c|}
\hline Ancho del talo $\sigma^{\lambda}$ & TAM & Largo de células epidermales & CELF \\
\hline Ancho del talo $q$ & TAF & 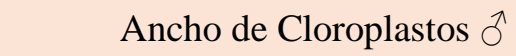 & CAM \\
\hline Largo del talo $\widehat{0}$ & TLM & Ancho de Cloroplastos $q$ & CAF \\
\hline Largo del talo $q$ & TLF & Largo de Cloroplastos $\hat{0}$ & CLM \\
\hline Largo del esporófito & $\mathbf{E L}$ & Largo de Cloroplastos 우 & CLF \\
\hline $\begin{array}{l}\text { Diámetro del } \\
\text { esporófito }\end{array}$ & EN & Ancho del anteridio & $\mathbf{A A}$ \\
\hline Largo del involucro & IN & Largo del anteridio & $\mathbf{A L}$ \\
\hline $\begin{array}{r}\text { Grosor de tejido } \\
\text { asimilativo }\end{array}$ & TA & Ancho del pedicelo del anteridio & PA \\
\hline $\begin{array}{r}\text { Ancho de células } \\
\text { interiores } \sigma^{-1}\end{array}$ & CIAM & Largo del pedicelo del anteridio & PL \\
\hline $\begin{array}{l}\text { Ancho de células } \\
\text { interiores } \\
\text { inter }\end{array}$ & CIAF & Ancho de las esporas & EA \\
\hline interiores 0 & CILM & ancho del estoma & ESA \\
\hline $\begin{array}{l}\text { Largo de células } \\
\text { interiores } \\
\text { Ancho de células }\end{array}$ & CILF & ancho del ostiolo & $\mathbf{O A}$ \\
\hline epidermales $\widehat{O}$ & CEAM & ancho de células del estoma & CEA \\
\hline $\begin{array}{l}\text { Ancho de células } \\
\text { epidermales } q\end{array}$ & CEAF & largo de células del estoma & CEL \\
\hline $\begin{array}{l}\text { Largo de células } \\
\text { epidermales } \delta^{\Uparrow}\end{array}$ & CELM & $\begin{array}{r}\text { Cantidad de anteridios por } \\
\text { cámara anteridial }\end{array}$ & $\mathbf{C A}$ \\
\hline
\end{tabular}

Tecnociencia, Vol. 23, $N^{\circ} 1$ 


\section{RESULTADOS}

Descripción morfológica de Leiosporoceros dussi.

Gametófito: Plantas dioicas de hábito taloso que crecen usualmente en colonias circulares que alcanzan los 11-17 cm de diámetro. Tomando en cuenta ambas poblaciones, el talo presenta una coloración que varía de verde claro, al estar adheridas al sustrato, a verde oscuro al ser desprendidas, hasta negro cuando secas; cuando las plantas se secan adheridas al sustrato en campo, el talo es rojizo (Fig. 1a). La forma del talo es alargada con ápice dicotómico, (1-) 2-3 (-4) cm de largo y (0.5) $1 \mathrm{~cm}$ de ancho, con margen entero a lobulado, lobos de $0.4-0.5 \mathrm{~cm}$ de ancho. Células del interior del talo en corte longitudinal alargadas, (57) 60-138 (-223) $\mu \mathrm{m}$ de largo y (20-) 45-52 (-75) $\mu \mathrm{m}$ de ancho, con forma rectangular a hexagonal. Células de la epidermis compuesta por células de (16- ) 27-30 (-55) $\mu \mathrm{m}$ de ancho y (22-) 41-47 (-80) $\mu \mathrm{m}$ de largo, papiloides, formando proyecciones superficiales en la epidermis (Fig. 1c-d). Cloroplastos en células epidermales, uno por célula, (8-) 15-16 (27) $\mu \mathrm{m}$ de ancho y (11-) 20 (-34) $\mu \mathrm{m}$ de largo, sin pirenoide. Los talos masculinos poseen cámaras anteridiales esféricas, de color amarillentas cuando maduras, irregularmente distribuidas desde la parte media, hasta el ápice del talo. Anteridios (21- ) 51 (-105) por cámara, con un ancho de (71-) 96 (-124) $\mu \mathrm{m}$ y (90-) 156 (-190) $\mu \mathrm{m}$ de largo; cubierta del anteridio con cuatro hileras de células. Pedúnculo de los anteridios de (16-) 24 (-34) $\mu \mathrm{m} \times(37-) 134$ (-220) $\mu \mathrm{m}$ con dos a tres células de ancho (Fig. 11a-d). Colonias de cianobacterias del género Nostoc dispuestas en la parte ventral del talo en canales ramificados, observándose como líneas verdes azulado a chocolatosas a lo largo de todo el talo (Fig. 2d).

Esporófito: Los talos femeninos presentan de $1-3$ esporófitos por individuo, desarrollándose hacia el ápice del talo (Fig. 10b). Cápsulas dehiscentes, de color verde a amarillo hasta chocolate cuando abiertas de (1.0-) $1.6(-2.5) \mathrm{cm}$. Involucro de la cápsula erecto y cilíndrico de (0.2-) $0.3(-0.4) \mathrm{cm}$ de largo (Cuadro 1). Valvas de la cápsula de (445- ) $576(-861) \mu \mathrm{m}$ de ancho (Fig. 10b). El tejido asimilativo presenta de 68 células en grosor ( $139-187 \mu \mathrm{m}$ de ancho). Estomas de la cápsula con (24- ) 51 (-104) $\mu \mathrm{m}$ de ancho, con poro de (4-) $11(-20) \mu \mathrm{m}$ de ancho y células protectoras de (12-) 21 (-48) $\mu \mathrm{m}$ de ancho y (48-) 75 (-93) $\mu \mathrm{m}$ 
de largo. Esporas amarillas, lisas sin ornamentación, con un diámetro ecuatorial de (19- ) 26 (-31) $\mu \mathrm{m}$ (Fig. 1f).

Fig. 1. Morfología del talo de $L$. dussii. A. Talo de $L$. dussii en descomposición. B. Colonia con plantas masculinas con cámaras anteridiales y plantas femeninas con esporofitos. C. Vista superficial del talo mostrando cloroplastos. D .Células papiloides (corte longitudinal del talo).
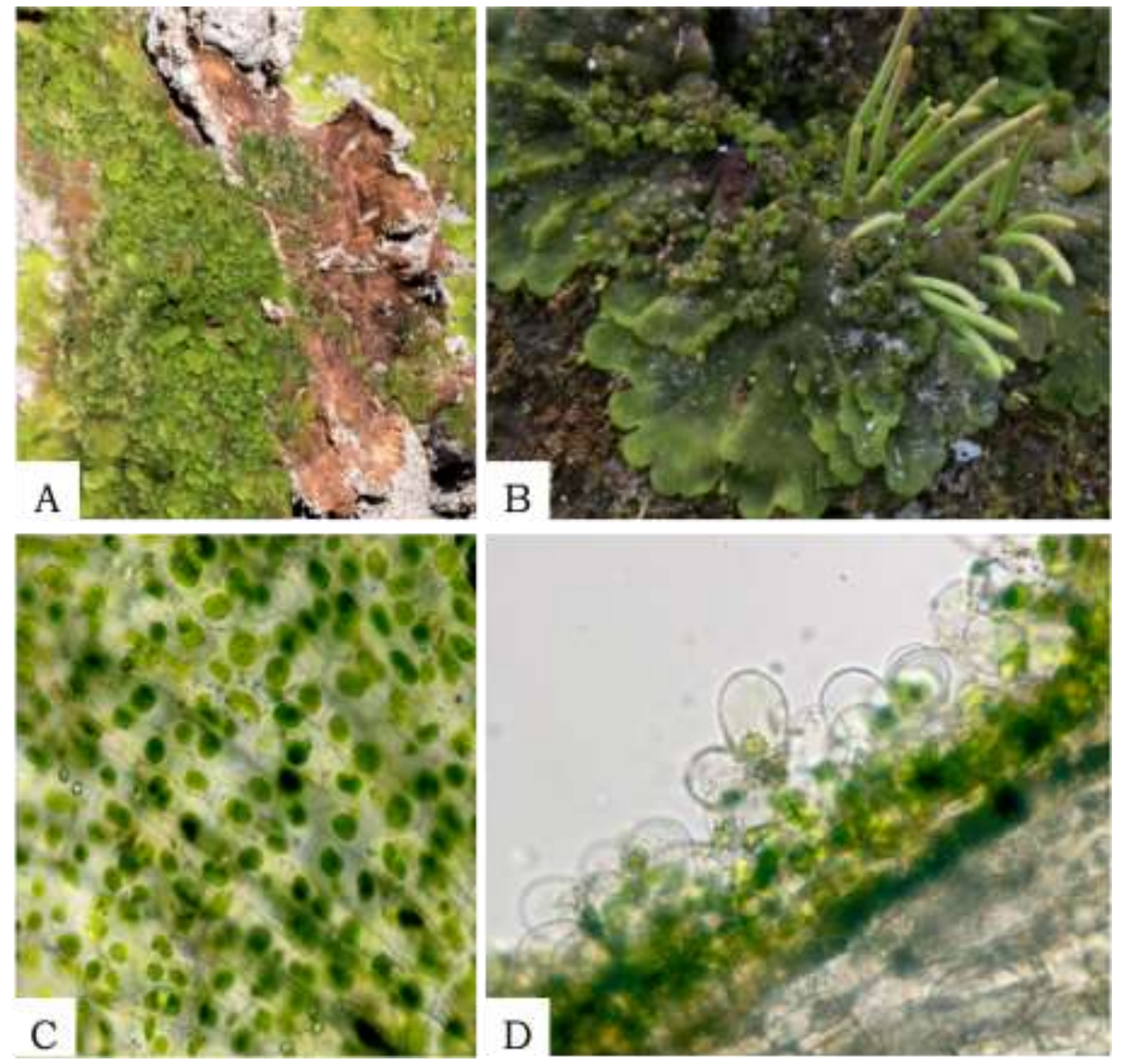
Fig. 2. Morfología del talo de L. dussii. A. Corte longitudinal de cámara anteridial mostrando anteridios en su interior. B. y C. Anteridios. D. Talo de planta masculina con cámaras anteridiales amarillas. E. Estomas presentes en el esporofito. F. Esporas

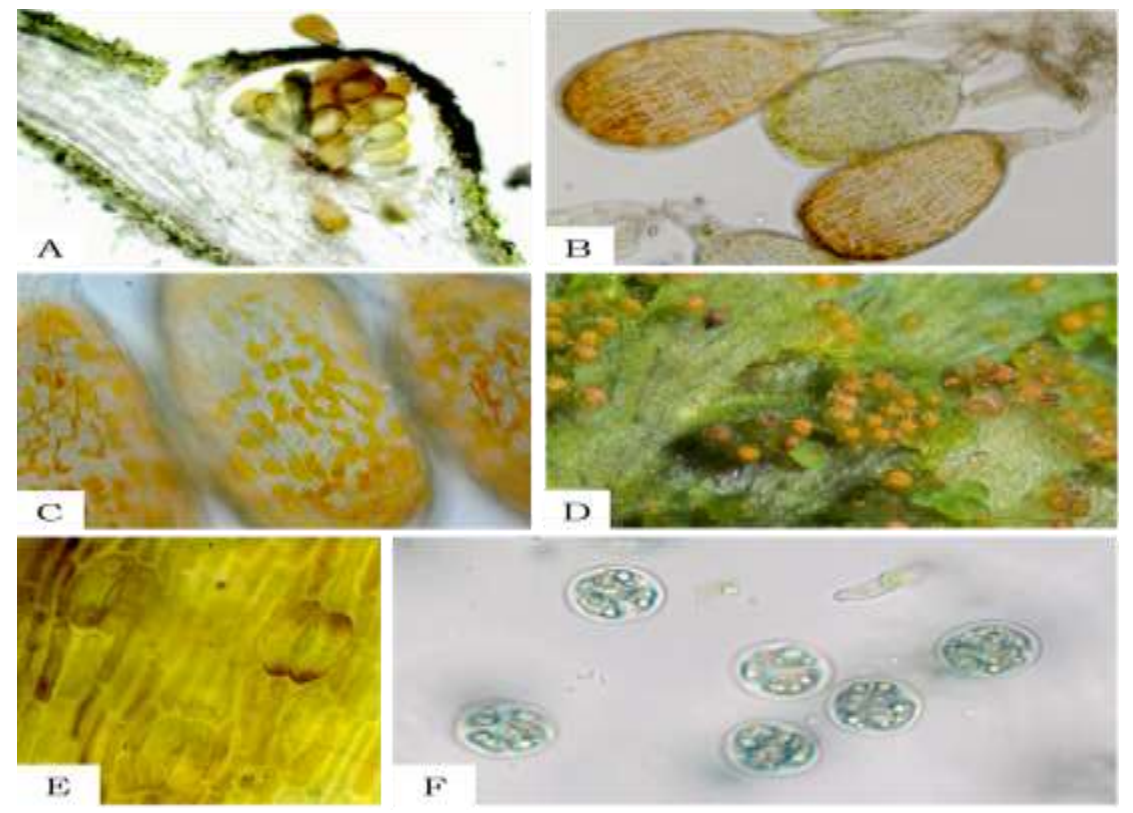

Comparación morfométrica de las poblaciones estudiadas

Los resultados obtenidos con la prueba multivariante MANOVA presentaron diferencias significativas en todos los grupos de variables entre las dos poblaciones estudiadas (Cuadro 3). La prueba univariante t-student mostró diferencias significativas individualmente entre cada variable estudiada solo en 15 de 22 tipos de medidas realizadas. Dentro de las 13 variables contempladas para la descripción del gametófito de L. dussii entre las dos poblaciones se obtuvieron diferencias morfométricas en 10 de estas variables. En cuanto a las nueve variables utilizadas para la descripción del esporófito se obtuvieron diferencias morfométricas en cinco de éstas. Las variables que presentaron diferencias significativas entre las dos poblaciones con la prueba $t$ student se resumen en el Cuadro 4. 
Cuadro 3. Análisis multivariante de datos. Esta tabla muestra los resultados con la prueba MANOVA obtenidos por cada grupo de variables. $\alpha=0.05$. Ver detalle de variables de cada grupo en el Cuadro 2.

\begin{tabular}{|l|r|r|r|r|c|}
\hline GRUPO & \multicolumn{5}{|c|}{ MANOVA } \\
\hline & \multicolumn{1}{|c|}{ Pillai } & approx F & \multicolumn{1}{c|}{ num Df } & den Df & $\operatorname{Pr}(>$ F) \\
\hline A & 0.9999 & 1278 & 8 & 1 & 0.02163 \\
\hline B & 0.54437 & 3.6839 & 12 & 37 & 0.001097 \\
\hline C & 0.24082 & 11.499 & 4 & 145 & $3.90 \mathrm{E}-08$ \\
\hline D & 0.39268 & 12.155 & 5 & 94 & $4.31 \mathrm{E}-09$ \\
\hline
\end{tabular}

Cuadro 4. Análisis univariante de datos. Se muestras los resultados de las variables morfométricas que presentaron diferencias significativas con las pruebas $t=$ student y Mann-Whitney, comparando las poblaciones por cada variable de manera independiente.

\begin{tabular}{|c|c|c|c|c|c|}
\hline \multirow[t]{2}{*}{ ESTRUCTURA } & \multicolumn{3}{|c|}{ POBLACIONES $\mu \mathrm{m}$} & \multirow[t]{2}{*}{ PRUEBA } & \multirow{2}{*}{$\begin{array}{l}p(\alpha= \\
0.05)\end{array}$} \\
\hline & $\begin{array}{l}\text { RÍO } \\
\text { GUAYABO }\end{array}$ & & RÍO INDIO & & \\
\hline \multicolumn{6}{|l|}{ GAMETÓFITO } \\
\hline Largo del talo $q$ & $\begin{array}{l}(0.7-) 1(-1.3) \\
\mathrm{cm}\end{array}$ & $<$ & $\begin{array}{l}(1-) 1.6(-2.0) \\
\mathrm{cm}\end{array}$ & $\mathrm{t}=-2.86$ & 0.021 \\
\hline $\begin{array}{l}\text { Ancho de células } \\
\text { epidermales } \not\end{array}$ & $(22-) 30(-39)$ & $>$ & $(17-) 25(-38)$ & $\mathrm{t}=2.81$ & 0.0069 \\
\hline $\begin{array}{l}\text { Ancho de células } \\
\text { epidermales } \sigma^{\lambda}\end{array}$ & $(21-) 34(-55)$ & $>$ & $(17-) 27(-43)$ & $\mathrm{t}=3.2$ & 0.0018 \\
\hline $\begin{array}{l}\text { Ancho de } \\
\text { cloroplastos } 9\end{array}$ & $(13-) 19(-28)$ & $<$ & $(9-) 25(-15)$ & $\mathrm{t}=2.91$ & 0.005 \\
\hline $\begin{array}{l}\text { Ancho de } \\
\text { cloroplastos }{ }^{\lambda}\end{array}$ & $(13-) 18(-25)$ & $>$ & $(8-) 14(-19)$ & $\mathrm{t}=2.089$ & 0.041 \\
\hline $\begin{array}{l}\text { Largo de } \\
\text { cloroplastos } 9\end{array}$ & $(14-) 22(-29)$ & $>$ & $(11-) 19(-28)$ & $\mathrm{t}=4.37$ & $\begin{array}{l}6.50 \mathrm{E}- \\
05\end{array}$ \\
\hline $\begin{array}{l}\text { Largo de } \\
\text { cloroplastos } \hat{0}\end{array}$ & $(14-) 22(-35)$ & $>$ & $(13-) 20(-28)$ & $\mathrm{t}=2.178$ & 0.0343 \\
\hline
\end{tabular}




\begin{tabular}{|l|l|l|l|l|l|}
\hline $\begin{array}{l}\text { Ancho del } \\
\text { pedúnculo del } \\
\text { anteridio }\end{array}$ & $(17-) 23(-30)$ & $<$ & $(15-) 26(-34)$ & $\mathrm{U}=1418$ & $\begin{array}{l}1.61 \mathrm{E}- \\
07\end{array}$ \\
\hline $\begin{array}{l}\text { Largo del } \\
\text { pedúnculo del } \\
\text { anteridio }\end{array}$ & $\begin{array}{l}(36-) 155(- \\
248)\end{array}$ & $>$ & $\begin{array}{l}(35-) 116(- \\
189)\end{array}$ & $\mathrm{t}=4.70$ & $\begin{array}{l}5.77 \mathrm{E}- \\
06\end{array}$ \\
\hline $\begin{array}{l}\text { Cantidad de } \\
\text { anteridios por } \\
\text { cámara anteridial }\end{array}$ & $(31-) 58(-105)$ & $>$ & $(21-) 44(-74)$ & $\mathrm{t}=2.22$ & 0.03 \\
\hline EPORÓFITO & & & & & \\
\hline $\begin{array}{l}\text { Ancho de las } \\
\text { esporas }\end{array}$ & $(19-) 24(-30)$ & $<$ & $(22-) 27(-31)$ & $\mathrm{t}=-4.866$ & $\begin{array}{l}4.35 \mathrm{E}- \\
06\end{array}$ \\
\hline $\begin{array}{l}\text { Ancho de los } \\
\text { estomas }\end{array}$ & $(37-) 58(-104)$ & $>$ & $(24-) 44(-57)$ & $\mathrm{t}=5.525$ & $\begin{array}{l}2.71 \mathrm{E}- \\
07\end{array}$ \\
\hline $\begin{array}{l}\text { Ancho del } \\
\text { ostiolo del } \\
\text { estoma }\end{array}$ & $(-8) 13(-21)$ & $>$ & $(4-) 10(-18)$ & $\mathrm{t}=4.668$ & $\begin{array}{l}9.64 \mathrm{E}- \\
06\end{array}$ \\
\hline $\begin{array}{l}\text { Ancho de células } \\
\text { protectoras de } \\
\text { los estomas }\end{array}$ & $(14-) 24(-48)$ & $>$ & $(12-) 18(-24)$ & $\mathrm{t}=3.45$ & $\begin{array}{l}8.19 \mathrm{E}- \\
04\end{array}$ \\
\hline $\begin{array}{l}\text { Largo de células } \\
\text { protectoras de } \\
\text { los estomas }\end{array}$ & $(65-) 78(-93)$ & $>$ & $(48-) 72(-90)$ & $\mathrm{U}=574$ & $\begin{array}{l}3.20 \mathrm{E}- \\
06\end{array}$ \\
\hline
\end{tabular}

\section{DISCUSIÓN}

Gametófito: Leiosporoceros dussii es dioico y forma colonias circulares de varios individuos que mueren desde la base, con diferentes etapas de desarrollo en la misma colonia (Villarreal \& Renzaglia 2006). Estas colonias a menudo están conformadas sólo por individuos masculinos o mezclados individuos masculinos y femeninos. La morfología del talo es variable en tamaño y forma, cuando jóvenes los talos muestran una forma alargada con ápice dicotómico con dimensiones de (1.8-) 2.7 ($3.5) \mathrm{cm}$ de largo en plantas femeninas y (1.7-) $2.2(-3.2) \mathrm{cm}$ de largo en plantas masculinas. A medida que la planta crece, la parte basal de ésta se desintegra quedando pequeñas rosetas que pueden medir de $0.8-1.7$ $\mathrm{cm}$ de largo en plantas femeninas, en las que se desarrolla el esporófito, y 0.7 - $1.0 \mathrm{~cm}$ de largo en plantas masculinas. Estos resultados concuerdan con la descripción de Hässel de Menéndez (1986), quien describe el talo de Leiosporoceros como un talo en forma de roseta de 
11-12 mm de ancho y borde lobado. Los talos jóvenes de las colonias de Leiosporoceros se encuentran en la periferia de la colonia. Otras especies de antocerotes como Anthoceros agrestis Paton y Phaeoceros coralinianus (Michx.) Prosk. muestran un crecimiento parecido, con fragmentación del talo en etapas avanzadas de desarrollo (Bissang 2003).

En la superficie del talo de Leiosporoceros se observaron múltiples células papiloides en la epidermis de ambos tipos de plantas. Estas son células papiloides, ya que no son proyecciones de las células, más bien es un grupo de células que se proyectan hacia el exterior del talo sobresaliendo de la capa de células epidermales. Estas células papiloides en la epidermis se observaron, tanto en plantas femeninas como en plantas masculinas. Este crecimiento irregular de células epidermales se ha reportado como excrecencias del talo (Hasegawa 1988) o crecimientos externos epidermales solo en plantas femeninas (Villarreal \& Renzaglia 2006). Las células papiloides, observadas en L. dussii, en vista superficial son de forma cuadrada a hexagonal, algunas veces rectangulares (Fig. 1c).

Los anteridios de L. dussii tienen forma ovalada, de color amarillento a naranja cuando maduros con un ancho de (71-) 96 (-124) $\mu \mathrm{m}$ y (90-) 156 $(-190) \mu \mathrm{m}$ de largo. Otros géneros de baja productividad de anteridios (1-6), como Phaeomegaceros R.J. Duff, J.C. Villarreal, Cargill \& Renzaglia, Dendroceros Nees, Megaceros Campb. y Nothoceros (R.M. Schust.) J. Haseg, presentan anteridios con dimensiones más grandes (García et al. 2012; Villarreal et al. 2007). Los anteridios de la población de Río Guayabo mostraron pedicelos más largos que la población de Río Indio, sin embargo, en la variable de ancho del pedicelo la población de Río Guayabo presentó pedicelos menos anchos que los de la población de Río Indio (Fig. 3). Los anteridios de L. dussii estudiados constan de un pedicelo de (16-) 24 (-34) $\mu \mathrm{m}$ de ancho y (37-) 134 (-220) $\mu \mathrm{m}$ de largo, pero en otros géneros de antocerotes de anteridios más grandes y con menos cantidad por cámara anteridial presentan pedicelos más cortos, por ejemplo, Notothylas (Cargill 2016) y Nothoceros (R.M. Schust.) J. Haseg. (Villarreal et al. 2007). 
Esporófito: Las plantas de L. dussii estudiadas presentaron de 1 a 3 esporófitos por planta. Las cápsulas de las plantas de L. dussii poseen un diámetro de (257-) 498 (-590) $\mu \mathrm{m}$ lo cual concuerda con la descripción de Hässel de Menéndez (1986), quien señala que estas pueden tener hasta $0.5 \mathrm{~mm}$ de diámetro. Las valvas de las cápsulas abiertas midieron de (445-) 576 (-861) $\mu \mathrm{m}$ de ancho. El involucro de $L$. dussii está cubierto de células papiloides al igual que la epidermis y midió hasta $3 \mathrm{~mm}$ de largo, un poco más largo de lo mencionado en la descripción de Hässel de Menéndez (1986) con involucros de hasta 2.5 $\mathrm{mm}$ de largo. El tejido asimilativo de los esporófitos de las plantas estudiadas midió 139 - $187 \mu \mathrm{m}$ de grosor, presentando de 6 - 8 capas de células.

Las esporas de las poblaciones de Leiosporoceros examinadas son de coloración amarilla y de forma ovaladas, pequeñas de unos (19-) 26 (31) $\mu \mathrm{m}$ y poseen una cubierta lisa sin ninguna clase de ornamentación, lo cual concuerda con lo mencionado descrito por Hässel de Menéndez (1986). En los antocerotes se han reportado tamaños de esporas desde $18 \mu \mathrm{m}$ en el género Leiosporoceros hasta más de $100 \mu \mathrm{m}$ en las esporas del género Dendroceros (Villarreal \& Renner 2013), siendo así uno de los antocerotes con esporas más pequeñas. La población de Río Indio posee esporas con dimensiones más grandes que las esporas de la población de Río Guayabo. Villarreal y Renner (2013), sugieren que el tamaño de las esporas podría estar ligado a la cantidad de esporas producidas, ya que producir esporas pequeñas implica un menor gasto energético y estas pueden ser producidas en un mayor número que las esporas grandes. De igual manera, es importante mencionar que, a pesar de presentar diferencias en el tamaño de las esporas y la cantidad de anteridios, Villarreal y Renner (2013) demostraron que no existe relación entre estas dos características en el grupo de los antocerotes.

Todas las medidas realizadas en los estomas mostraron diferencias al comparar las dos poblaciones. La población de río Guayabo mostró estomas más grandes que la población de Río Indio. Esta diferencia en las dimensiones de los estomas puede ser debido a variaciones en las condiciones ambientales de los dos sitios de estudios, ya que la población de Río Guayabo crece en áreas boscosas y en la población de Río Indio ocurre en un área abierta, expuesta al sol, lo que provoca un microclima más seco. El ancho de los estomas en Leiosporoceros fue de (-24) $51(-104) \mu \mathrm{m}$ y las células estomáticas son de $75(-93) \mu \mathrm{m} \times 21$ (48) $\mu \mathrm{m}$. De acuerdo con algunos estudios, L. dussii es uno de los 
antocerotes con células estomáticas más grandes junto con Phymatoceros bulbiculosus (Brot.) Stotler, W.T. Doyle \& Crand. -Stotl. $(66.8 \mu \mathrm{m})$ y Phaeoceros engelii Cargill \& Fuhrer $(81.3 \mu \mathrm{m})$ (Renzaglia et al. 2017).

Comparación morfométrica de las poblaciones estudiadas: A pesar de que en el grupo A de variables morfométricas solo se obtuvo diferencias en la variable de largo del talo de plantas femeninas comparando entre las dos poblaciones en las pruebas de análisis independiente, el PCA de este grupo muestra una marcada diferencia de las dos poblaciones, especialmente en las variables de largo y ancho del talo separando de igual manera a las plantas por género (Fig. 4). Las variables contempladas en el Grupo B, correspondientes a las células del talo y los cloroplastos presentaron diferencias entre ellas, las cuales se muestran en la una marcada separación en la gráfica de biplot entre plantas femeninas y masculinas (Fig. 5). En las pruebas variables independientes se obtuvo diferencia específicamente en la variable ancho de células epidermales, en donde las plantas de la población de río Guayabo tuvieron células epidermales más anchas que las plantas de la población de Río Indio, tanto en plantas masculinas como femeninas. Los cloroplastos de L. dussii ocupan al menos la mitad del volumen celular. El tamaño de los cloroplastos de ambos tipos de plantas, masculinas y femeninas, estuvo entre un promedio de $20-21 \mu \mathrm{m}$. Según los resultados obtenidos, las plantas de la población de río Guayabo poseen cloroplastos más grandes que los de la población de Río Indio. Esta diferencia en el tamaño de los cloroplastos podría deberse, probablemente, a diferencias en la disponibilidad de luz solar en las dos poblaciones. El grupo $\mathrm{C}$ solo mostró diferencias en la cantidad de anteridios (Fig., 3 y 6). El PCA del grupo D muestra a la población de río Guayabo divido en dos grupos, uno relacionado con la población de Río Indio y otro totalmente separado, mostrando una marcada diferencia entre las dimensiones de los estomas dentro de la población de Río Guayabo (Fig. 7). 
Fig. 3. Comparación de la cantidad de anteridios entre las dos poblaciones. RG: Río Guayabo. RI: Río Indio. ( $\mathrm{p}=0.03, \alpha=0.05)$

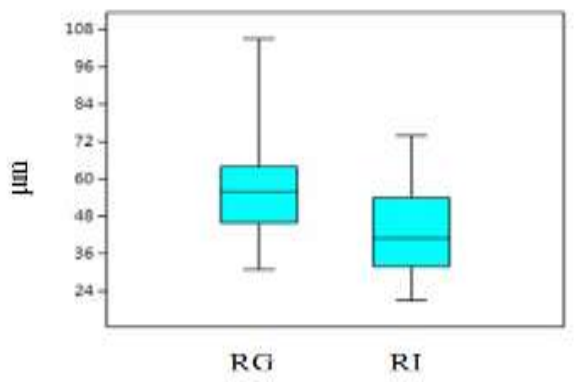

Fig. 4. Biplot de Análisis de Componentes Principales del Grupo A de Variables Morfométricas Comparando Las Dos Poblaciones Y Los Tipos De Plantas. Puntos: Población de Río Indio. Triángulos: Población de Río Guayabo. En rojo se agrupan las poblaciones estudiadas y el amarillo el género de las plantas. Ver detalle de variables en el Cuadro 2

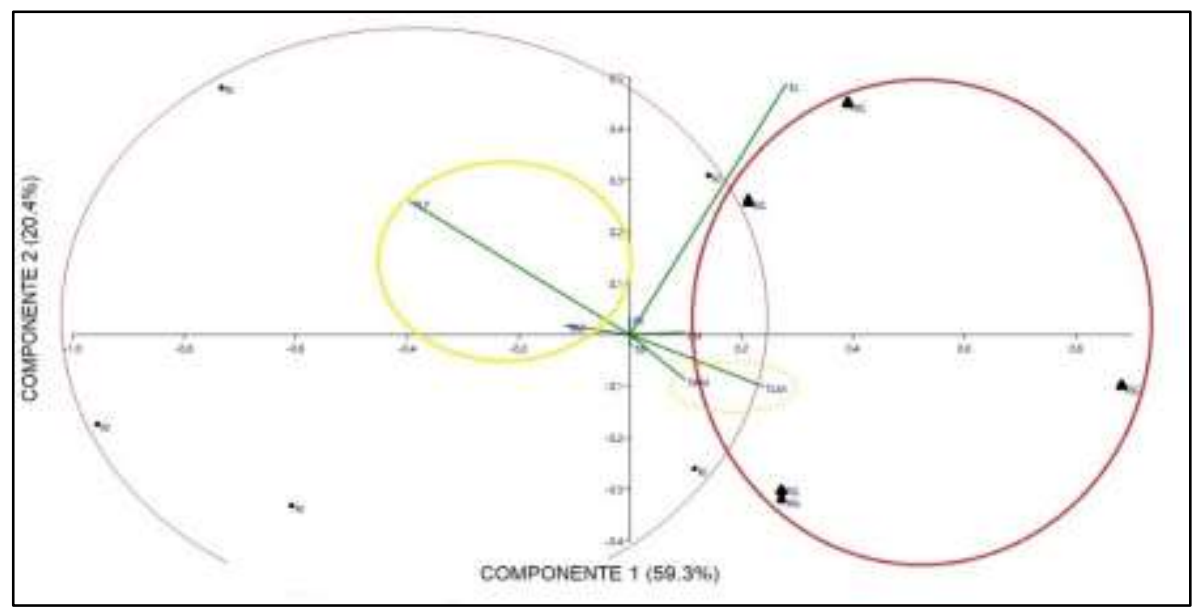


Fig. 5. Biplot de Análisis de Componentes Principales del Grupo B de Variables Morfométricas Comparando Las Dos Poblaciones Y Los Tipos De Plantas. Puntos: Población de Río Indio. Triángulos: Población de Río Guayabo. En rojo se agrupan las poblaciones estudiadas y el amarillo el género de las plantas. Ver detalle de variables en el Cuadro 2

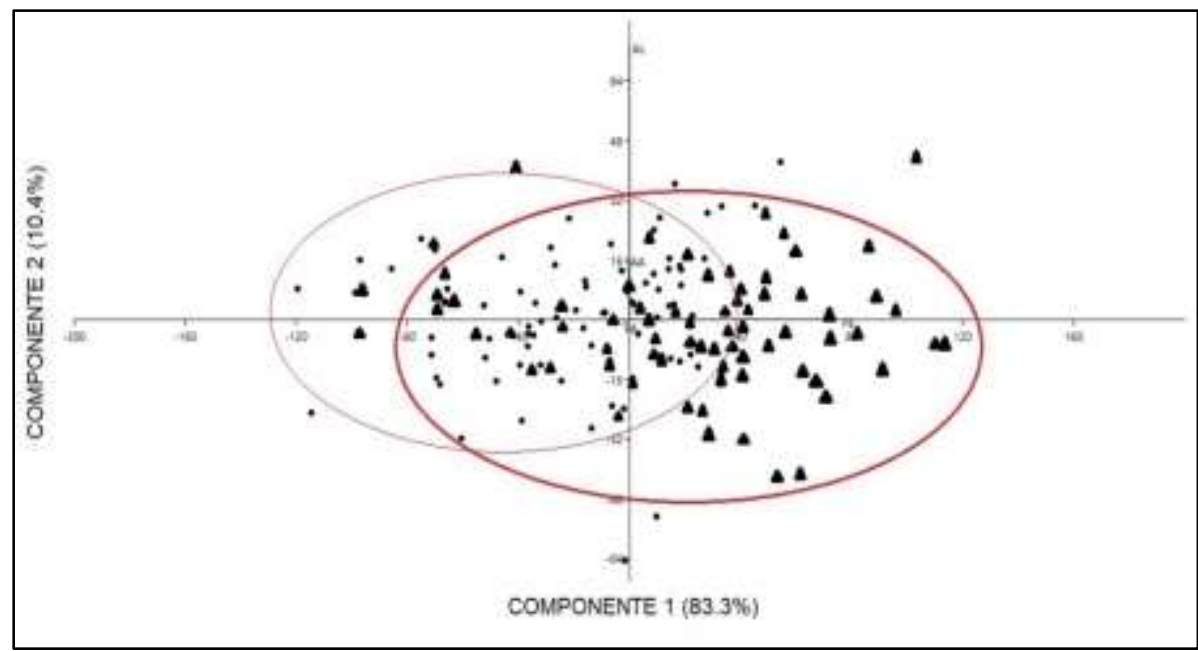

Fig. 6. Biplot de Análisis de Componentes Principales del Grupo C de Variables Morfométricas Comparando Las Poblaciones Estudiadas. Puntos: Población de Río Indio. Triángulos: Población de Río Guayabo. Se agrupan las poblaciones en rojo. Ver detalle de variables en el Cuadro 2

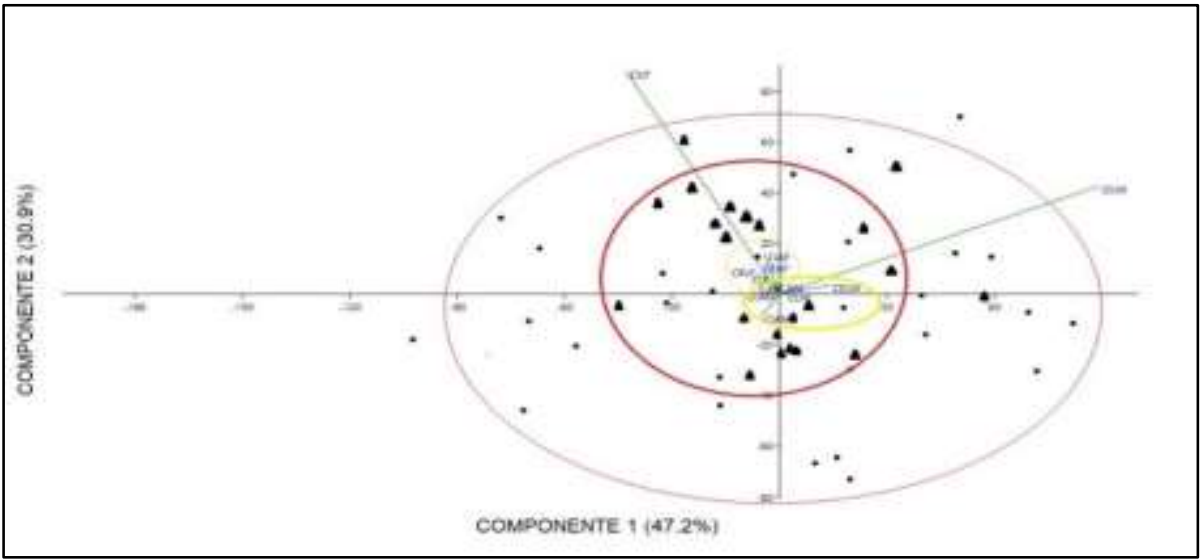

Tecnociencia, Vol. 23, $N^{\circ} 1$ 
Fig. 7. Biplot de Análisis de Componentes Principales del Grupo D de Variables Morfométricas Comparando Las Poblaciones Estudiadas. Puntos: Población de Río Indio. Triángulos: Población de Río Guayabo. Se agrupan las poblaciones en rojo. Ver detalle de variables en el Cuadro 2

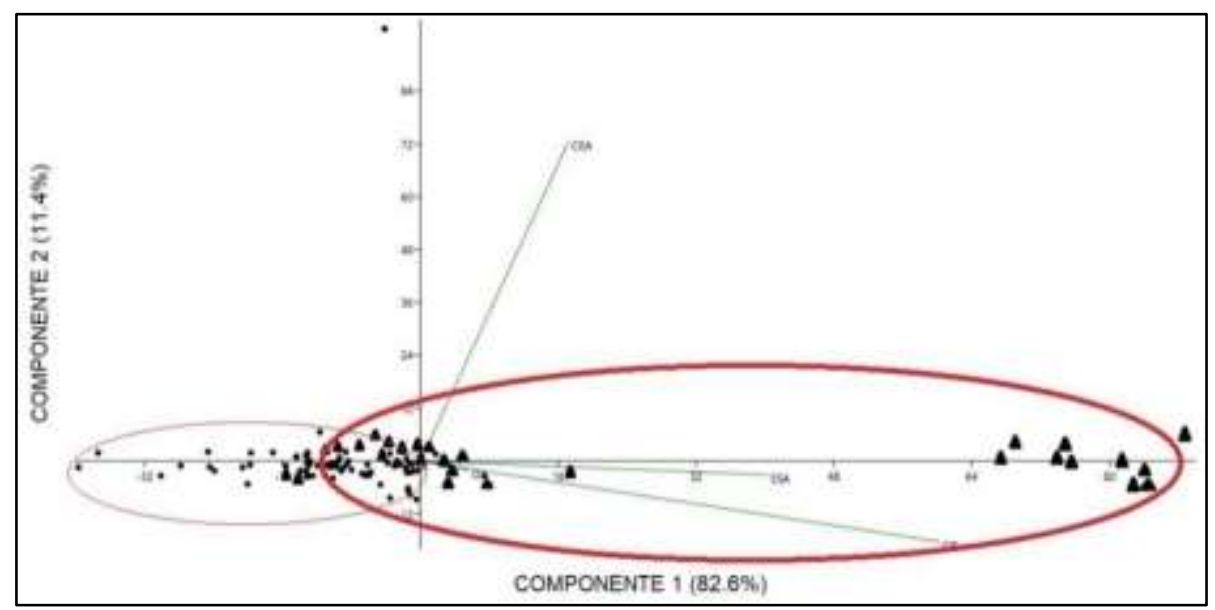

\section{CONCLUSIÓN}

Las dos poblaciones de L. dussii estudiadas presentaron diferencias comparando las variables morfométricas según las poblaciones y entre plantas masculinas y femeninas. No se presentaron suficientes evidencias para sugerir que estas diferencias sean debido a variaciones climáticas presentes en las poblaciones, por lo que se recomienda realizar estudios más extensos que incluyan otras poblaciones e integrar múltiples variables ambientales.

\section{AGRADECIMIENTOS}

Este proyecto fue apoyado por la Secretaría Nacional de Tecnología y Ciencias (SENACYT) y el Instituto de Investigaciones Tropicales Smithsonian (STRI). Se agradece a la Dra. Noris Salazar y al Lic. José Gudiño por su apoyo en el desarrollo de este proyecto. A Maycol Madrid, Keri Greig y Zarluis Mijango por su ayuda con los análisis estadísticos. 


\section{REFERENCIAS}

Bissang, I. (2003). Population development, demographic structure, and life cycle aspects of two hornwort in Switzerland. Lindbergia 28:105112. http://www.jstor.org/stable/20150137 .

de Menéndez, G. G. H. (1986). Leiosporoceros Hässel n. gen. and Leiosporocerotaceae Hässel $\mathrm{n}$. fame of Anthocerotopsida. Journal of bryology 14: 255-259. https://doi.org/10.1179/jbr.1986.14.2.255

Evert, RF (2006). Anatomía vegetal de Esaú: meristemos, células y tejidos del cuerpo vegetal: su estructura, función y desarrollo. John Wiley \& Sons.

Garcia, C., Sérgio, C., Villarreal, J. C., Sim-Sim, M., \& Lara, F. (2012). The hornworts Dendroceros Nees and Megaceros Campb. in São Tomé e Príncipe (Africa, Gulf of Guinea) with the description of Dendroceros paivae sp. nov. Cryptogamie, Bryologie, 33: 3-21. https://doi.org/10.7872/cryb.v33.iss1.2012.003

Hasegawa, J. (1988). A proposal for a new system of the Anthocerotae, with a revision of the genera. Journal of the Hattori Botanical Laboratory, 64: 87-95.

Kenrick, P. \& Crane, P. R. (1997). The origin and early evolution of plants on land. Nature, 389: 33-39. https://ci.nii.ac.jp/naid/40005331350

Konrat, M., Shaw, J. \& Renzaglia, K. S. (2010). A special issue of Phytotaxa dedicated to Bryophytes: The closest living. Phytotaxa 9: 510.https://www.biotaxa.org/Phytotaxa/article/viewFile/phytotaxa.9.1.3/ 8692

Magill, R. (2010). Moss Diversity: new look at old numbers. Phytotaxa 9: 167-174. https://doi.org/10.11646/phytotaxa.9.1.9

One Thousand Plant Transcriptomes Initiative. (2019). One thousand plant transcriptomes and the phylogenomics of green plants. Nature, 574(7780), 679. https://www.nature.com/articles/s41586-019-1693-2 
Pressel, S., Goral, T. \& Duckett, J. G. (2014). Stomatal differentiation and abnormal stomata in hornworts. Journal of Bryology, 36: 87-103. https://doi.org/10.1179/1743282014Y.0000000103

Renzaglia, K. S., Villarreal, J. C., Piatkowski, B. T., Lucas, J. R., \& Merced, A. (2017). Hornwort stomata: architecture and fate shared with 400-million-year-old fossil plants without leaves. Plant Physiology, 174(2), 788-797. https://doi.org/10.1104/pp.17.00156

Stephani, F. (1893). Hepaticarum Species novae. II. Hedwigia 32, 137147.

Stephani, F. (1912-1917). Species Hepaticarum, 5, Geneve et Bale.

Team, R. C. (2018). R v3. 5.1 R: a language and environment for statistical computing. R Core Team, Vienna, Austria.

Vaughn, K. C., Campbell, E. O., Hasegawa, J., Owen, H. A. \& Renzaglia, K. S. (1990). The pyrenoid is the site of ribulose 1, 5bisphosphate carboxylase/oxygenase accumulation in the hornwort (Bryophyta: Anthocerotae) chloroplast. Protoplasma. 156, 117. https://doi.org/10.1007/BF01560650

Villarreal, J.C. \& Renzaglia, K.S. (2006). Structure and development of Nostoc strands in Leiosporoceros dussii (Anthocerotophyta): A novel symbiosis in land plants. American Journal of Botany. 93, 693-705. https://doi.org/10.3732/ajb.93.5.693

Villarreal, J. C., Menéndez, G. H. D., \& Allen, N. S. (2007). Nothoceros superbus (Dendrocerotaceae), a new hornwort from Costa Rica. The Bryologist. 110, 279-285. https: // www. jstor .org / stable / 20110857

Villarreal, J. C., Cargill, C., Hagborg, A., Söderström, L. \& Renzaglia, K. (2010). A synthesis of hornwort diversity: Patterns, causes and future work. Phytotaxa. 9, 150-166. https://doi.org/10.11646/phytotaxa.9.1.8

Villarreal, JC, Renner, SS Correlación de monoicidad y dióicidad en hornworts, el grupo hermano aparente de las plantas vasculares. BMC Evol Biol 13, 239 (2013). https://doi.org/10.1186/1471-2148-13-239 
Villarreal, J.C. \& Renzaglia, K. S. (2015). The hornworts: important advancements in early land plant evolution. Journal of Bryology. 37: 3, 157-170. https://doi.org/10.1179/1743282015Y.0000000016

Von Konrat, M., Soderstrom, L., Renner, M. A., Hagborg, A., Briscoe, L. \& Engel, J. J. (2014). Early Land Plants Today (ELPT): How many liverwort species are there? Phytotaxa. 9, 22-40. https://doi.org/10.11646/phytotaxa.9.1.5 